\title{
Paraneoplastic Cushing's syndrome associated with bronchopulmonary carcinoid tumor in youth: A case report and review of the literature
}

\author{
WEN-YA LI ${ }^{1}$, XU-DONG LIU ${ }^{2}$, WEI-NAN LI ${ }^{3}$, SI-YUAN DONG ${ }^{1}$, XIAO-HAN QU ${ }^{1}$, \\ SHU-LEI GONG ${ }^{1}$, MING-RUI SHAO ${ }^{1}$ and LIN ZHANG ${ }^{1}$ \\ Departments of ${ }^{1}$ Thoracic Surgery, ${ }^{2}$ Rheumatology and Immunology, and ${ }^{3}$ Pathology, \\ The First Affiliated Hospital of China Medical University, Shenyang, Liaoning 110001, P.R. China
}

Received March 27, 2015; Accepted April 22, 2016

DOI: $10.3892 / 01.2016 .4572$

\begin{abstract}
Paraneoplastic Cushing's syndrome (CushingPS) caused by bronchopulmonary carcinoid tumors presents a diagnostic challenge for clinicians. The present study reports the case of an 18-year-old male patient presenting with rapid weight gain, polyuria, polydipsia and progressive muscle weakness. Chemical and imaging findings suggested ectopic secretion of adrenocorticotropin. Whole-body ${ }^{18}$ fluorine-fluorodeoxyglucose $\left({ }^{18} \mathrm{FDG}-\mathrm{PET} / \mathrm{CT}\right)$ positron-emission tomography revealed an increased uptake of ${ }^{18} \mathrm{FDG}-\mathrm{PET} / \mathrm{CT}$ in the right middle lung mass and lobar lymph node. Postoperative pathology confirmed the presence of a typical carcinoid, as well as a lobar lymph node metastasis. The patient underwent a right middle lobectomy with mediastinal lymph node resection, which resulted in symptom clearance, followed by rapid weight loss. No CushingPS or tumor recurrence was observed at the 3-month postoperative follow-up.
\end{abstract}

\section{Introduction}

Paraneoplastic Cushing's syndrome (CushingPS) has been attributed to ectopic adrenocorticotropin (ACTH) secretion (EAS), which may result in several types of tumors, including carcinoid and pancreatic neuroendocrine tumors, small cell lung cancer and thyroid medullary carcinoma (1). The bronchopulmonary carcinoid tumor (BCT) accounts for 1-2\% of all adult malignancies of the lung and $20-30 \%$ of all carcinoid tumors (2-5).

Based on the World Health Organization classification, BCTs are divided into typical carcinoids, which are low-grade

Correspondence to: Dr Lin Zhang, Department of Thoracic Surgery, The First Affiliated Hospital of China Medical University, 155 North Nanjing Street, Shenyang, Liaoning 110001, P.R. China E-mail: 41578203@qq.com

Key words: ectopic adrenocorticotropin, paraneoplastic Cushing's syndrome, bronchopulmonary carcinoid tumor, lobar lymph node metastasis, ${ }^{18}$ fluorine-fluorodeoxyglucose tumors with a low mitotic rate, and atypical carcinoids, which are intermediate-grade tumors with a higher mitotic rate and/or necrosis. Typical carcinoids are approximately four times more common than atypical carcinoids. The worldwide incidence of BCT is $0.2-2$ per 100,000 individuals annually, with the majority of studies reporting a higher incidence in women compared with men, and in Caucasian compared with African-American individuals (2-4,6-8). Several reports have suggested that the incidence of BCT is increasing $(3,4,8)$; however, this may be partly due to the increased use of advanced medical imaging techniques that are more sensitive in detecting asymptomatic tumors.

In general, BCTs are considered to be low- to moderate-grade malignant tumors; however, when associated with CushingPS, they are clinically aggressive (9). The present study reports a rare case of CushingPS in a teenager who was diagnosed with BCT and lymphadenopathy.

\section{Case report}

In September 2014, an 18-year-old man visited the Department of Endocrinology, The First Affiliated Hospital of China Medical University, Liaoning, presenting with a 3-month history of rapid weight gain $(15 \mathrm{~kg})$ characterized by central obesity involving the trunk and face with sparing of the limbs. The patient additionally complained of polyuria, polydipsia and progressive muscle weakness. The patient's height was $167 \mathrm{~cm}$ and his weight was $83 \mathrm{~kg}$. The patient's blood pressure was $160 / 100 \mathrm{mmHg}$ at presentation. Physical examination revealed typical Cushingoid features, including fat pads along the collarbone, back of the neck (buffalo hump) and face (moon face), along with generalized purple striae and hair growth on the back and both arms.

Routine laboratory test results revealed the following: Leukocytosis $\left(11.4 \times 10^{9} / 1\right)$ with neutrophilia (81.2\%); hyperglycemia (11.2 mmol/1); hypokalemia (2.54 mmol/1); metabolic alkalosis (pH 7.56); base excess, $6.8 \mathrm{mmol} / \mathrm{l}$; and standard $\mathrm{HCO}^{3-}, 31.7 \mathrm{mmol} / \mathrm{l}$. A marked elevation in serum cortisol (00:00 h, 1,397.0 nmol/1; 08:00 h, 1,750.0 nmol/1; 15:00 h, 1,566.0 nmol/l) and АCTH (00:00 h, $149.8 \mathrm{pg} / \mathrm{ml} ; 08: 00 \mathrm{~h}$, $211.0 \mathrm{pg} / \mathrm{ml} ; 15: 00 \mathrm{~h}, 198.6 \mathrm{pg} / \mathrm{ml})$ levels was also observed, without diurnal rhythm. Additional examinations revealed no 

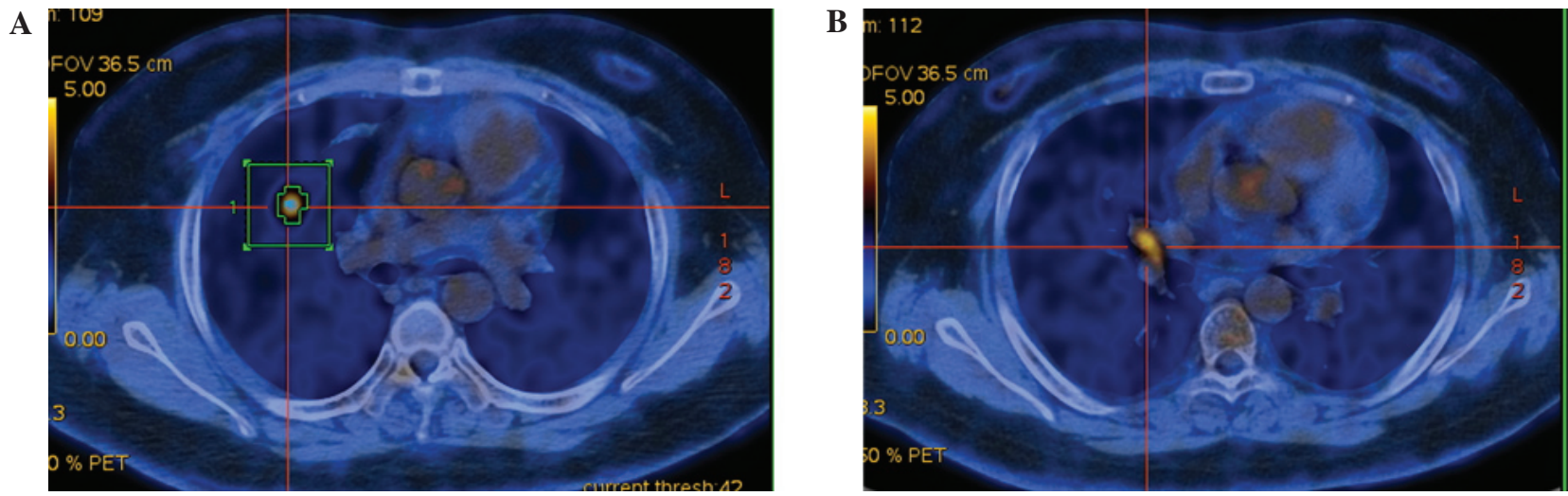

Figure $1 .{ }^{18} \mathrm{~F}$-FDG positron-emission tomography scan showing high ${ }^{18} \mathrm{~F}-\mathrm{FDG}$ uptake in (A) a right middle lobe nodule and (B) a lobar lymph node. ${ }^{18} \mathrm{~F}-\mathrm{FDG}$, ${ }^{18}$ fluorine-fluorodeoxyglucose.

A

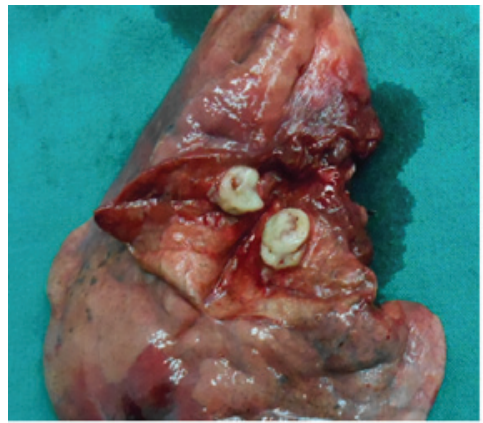

B

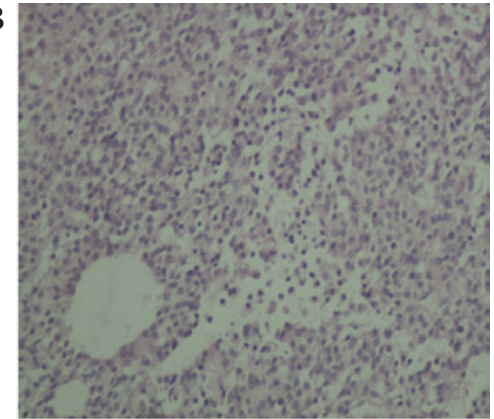

C

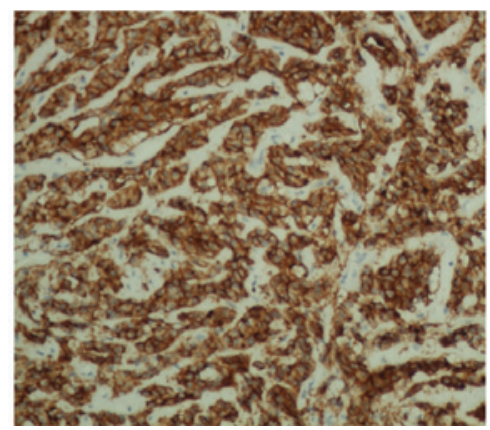

Figure 2. (A) Gross pathology of the carcinoid tumor revealed a grey-white mass with clear boundaries measuring $1.2 \mathrm{~cm}$ in diameter. (B) Microscopic examination of the specimen showed a typical carcinoid tumor (hematoxylin and eosin staining; magnification, x200). (C) Immunohistochemical staining showed positivity of the tumor cells for adrenocorticotropin (magnification, x200).

response to either low- or high-dose dexamethasone suppression. Based on the aforementioned findings, CushingPS due to EAS was initially suspected. Abdominal computed tomography (CT) and brain magnetic resonance imaging (MRI) scans revealed no abnormalities in the adrenal and pituitary glands. A chest CT scan revealed a mass in the right middle lung measuring $1.2 \mathrm{~cm}$ in diameter, which was evidenced by bilateral and diffusely scattered micronodular shadows. Whole-body ${ }^{18}$ fluorine-fluorodeoxyglucose positron-emission tomography/computed tomography $\left({ }^{18} \mathrm{~F}-\mathrm{FDG}\right.$ PET/CT $)$ confirmed a high ${ }^{18} \mathrm{~F}$-FDG uptake in the right middle lung [maximum standard uptake value, $\left(\mathrm{SUV}_{\max }\right), 5.68$ ] and lobar lymph node $\left(\mathrm{SUV}_{\max }, 4.89\right.$; Fig. 1), which supported the clinical diagnosis of CushingPS associated with right middle lung cancer.

The patient was subsequently treated with ketoconazole $(1,000 \mathrm{mg} /$ day $)$ and intravenous potassium supplementation for 1 week. Following the decrease of the plasma cortisol levels, the middle lobe was resected with hilar and mediastinal lymph node dissection.

Tissue specimens were fixed with $10 \%$ neutral formalin and embedded in paraffin, and $4-\mu \mathrm{m}$-thick sections were prepared. Immunostaining was performed using the avidinbiotin-peroxidase complex method (Ultrasensitive ${ }^{\mathrm{TM}}$, MaiXin, Fuzhou, China). Paraffin sections were dewaxed in xylene and rehydrated in graded alcohols. Antigen retrieval was performed by heating the sections for $1.5 \mathrm{~min}$ in $0.01 \mathrm{~mol} / \mathrm{L}$ citrate buffer, $\mathrm{pH}$ 6.0. Non-specific staining was reduced by incubation in blocking buffer containing goat serum (SP KIT-B1; MaixinBio, Fuzhou, China) for $30 \mathrm{~min}$. Then, the sections were incubated with ACTH antibody (rabbit polyclonal to ACTH, dilution, 1/200, cat no. ab74976, Abcam) overnight at $4^{\circ} \mathrm{C}$. The following day, the sections were incubated with appropriate secondary antibodies for $30 \mathrm{~min}$. The reaction was visualized 

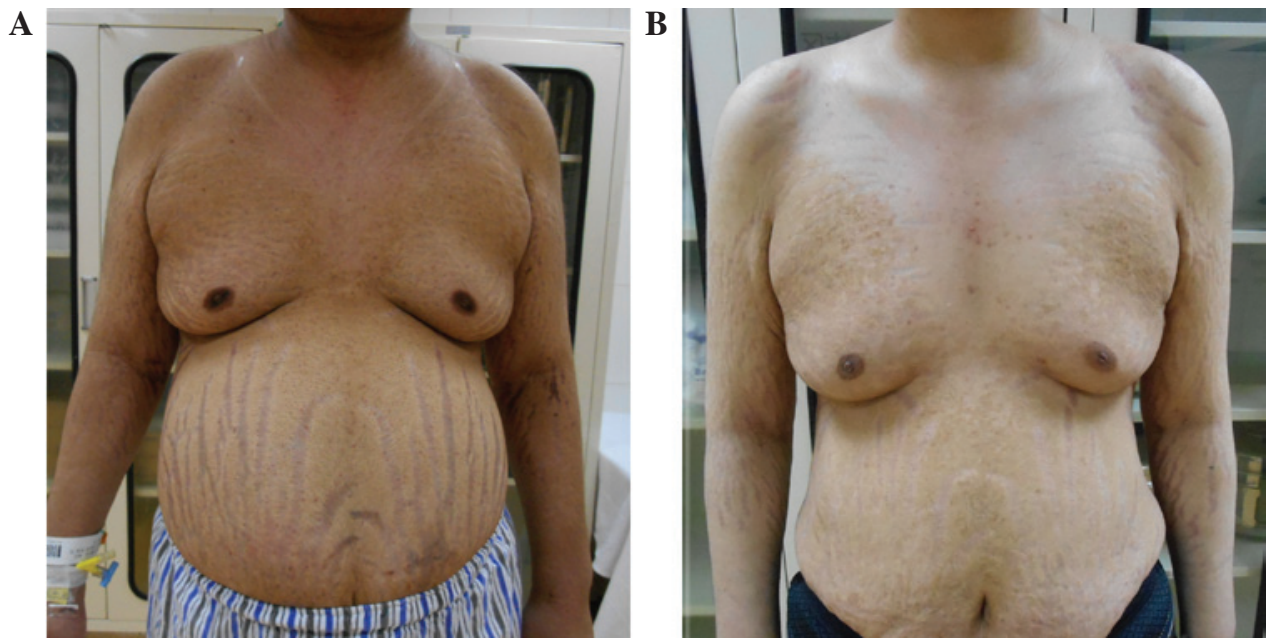

Figure 3. Patient with paraneoplastic Cushing's syndrome (A) upon presentation and (B) at the 3-month postoperative follow-up.

using DAB (DAB-0031; Maixin-Bio) plus chromogen. Specimens were examined using a BX50 microscope (Olympus Corp. Tokyo, Japan). For serum controls, $1 \%$ BSA in PBS was used in place of the primary antibody as a negative control. Pathology indicated a typical carcinoid tumor with lobar lymph node metastasis, staged as IIA (T1aN1M0). Immunohistochemistry additionally revealed that the tumor was positive for ACTH (Fig. 2). Symptoms were alleviated following surgery. Blood pressure, plasma glucose, potassium, cortisol, ACTH and 24-h urinary cortisol were normalized within a week. The postoperative recovery was uneventful, and the patient was discharged 14 days after the surgery. No CushingPS or tumor recurrence was noted at the 3-month postoperative follow-up. In addition, the patient had lost $13 \mathrm{~kg}$ (Fig. 3). Postoperative chemotherapy was administrated for four courses (Docetaxel $75 \mathrm{mg} / \mathrm{m}^{2}$ and Cisplatin $75 \mathrm{mg} / \mathrm{m}^{2}$ ), and the patient reached 15-months follow-up with no CushingPS or tumor recurrence.

\section{Discussion}

CushingPS due to BCT is a rare clinical occurrence, which has been reported in only a few case reports and case series. In fact, to the best of our knowledge, only four published surgical studies have reported data from $>5$ patients (10-13). According to a report of 19 BCT-induced CushingPS cases, the mean age of the patients was observed to be 43 years (11). Terzolo et al (12) reported 14 cases of EAS associated with a variety of tumor types of patients aged 26-76 years (mean age 53 years), with CushingPS being the predominant diagnosis in patients of advanced age, suggesting an increased occurrence of CushingPS in elderly compared with young individuals. However, sporadic cases of CushingPS in young patients have additionally been reported. Arioglu et al (13) reported a case of CushingPS in a 9-year-old girl, which was attributed to ectopic hormone secretion. The present study reported a case of CushingPS secondary to BCT in a teenager, which suggested an early onset of the syndrome.

EAS is difficult to diagnose and accurately localize. Symptoms typically manifest 6-84 months ahead of the diagnosis, with the average time to diagnosis ranging up to 24 months (14). In $12-19 \%$ of patients, the source of the tumor is unknown $(15,16)$. Imaging modalities, including CT and MRI, lack the sensitivity and specificity required to identify an ectopic ACTH lesion (16). ${ }^{111}$ Indium-labeled octreotide scintigraphy $\left({ }^{111}\right.$ In-OCT) has been reported as an effective diagnostic modality, as $80 \%$ of BCTs express receptors for somatostatin (17). ${ }^{111}$ In-OCT is considered helpful in the localization of EAS tumors. In addition, it may enable rapid, non-invasive differential diagnosis between ACTH-secreting pituitary adenomas and malignancies caused by EAS, followed by radioguided surgery in the treatment of bronchial carcinoid (18). ${ }^{18}$ FDG-PET and ${ }^{18}$ F-Fluorine-18-1-dihydroxyphenylalanine $\left({ }^{18} \mathrm{~F}\right.$-DOPA)-PET scans should also be considered as a secondary diagnostic tool for early diagnosis of EAS when conventional imagery fails to detect the presence of tumors (19). FDG-PET has a sensitivity of $64 \%$ and a positive predictive value of $53 \%$ in the identification of EAS (20). In the present case, ${ }^{18} \mathrm{~F}$-FDG PET indicated an EAS tumor and a metastatic lymph node; the patient underwent radical resection, which led to the amelioration of the clinical symptoms.

Surgery is considered to be one of the preferred treatment options for localized ectopic lesions, and leads to a reduction in cortisol levels (17). In the present case, a right middle lobectomy with mediastinal lymph node resection was selected based on the pathological diagnosis of a typical carcinoid and lobar lymph node metastasis (stage N1). As reported, $87 \%$ of typical carcinoids do not present with lymph node involvement. Combined with CushingPS, a higher rate of lymph node metastasis and postoperative local recurrence rate was found $(10,21)$. These findings indicated that BCT with CushingPS is an aggressive clinical entity. Lobectomy combined with complete lymphadenectomy is recommended for carcinoid tumors associated with CushingPS $(10,14,22)$; otherwise, an occult residual metastatic lymph node may present, leading to persistent or recurrent CushingPS (11).

Patients with CushingPS are at increased risk of life-threatening infection and venous thromboembolism (23); however, due to their resistance to cytotoxic agents and radiation (10), such patients generally have a poor prognosis. In a clinical study conducted by the MD Anderson Cancer Center, 82\% of patients with CushingPS caused by small-cell lung cancer succumbed to their condition within 2 weeks of the initiation 
of chemotherapy. Rectifying the patients' biochemical profile over a period of a few weeks prior to chemotherapy markedly improved the prognosis (24). Regulating the production of cortisol drastically alleviated the complications. Ketoconazole (200-1,200 mg/day) has been shown to inhibit the enzymes 11-hydroxylase and 17-hydroxylase in the cortisol biosynthesis pathway, and has been widely used due to its rapid mechanism of action and low frequency of side-effects (25). The present study demonstrated that ketoconazole combined with potassium supplementation was able to effectively control the preoperative hormone and ion levels of the patient. Ketoconazole treatment resulted in the biochemical and hormonal improvement of the patient with EAS; however, this drug may impair the cortisol response to stress; therefore, replacement corticosteroids should be considered for patients that exhibit a hormonal response, and use of moderate- to high-dose corticosteroids is recommended for any potential stress situations. Successful treatment of the underlying tumor is key to controlling the syndrome (26). A diminished objective response and sensitivity to first-line therapy and lack of a response to second-line therapy are indicators of the necessity to initiate palliative care early at first line and exclusively at relapse (27).

In conclusion, the present study reported a rare case of CushingPS in a teenager with BCT and N1 lymph node metastasis. The present findings suggested that BCT associated with CushingPS may be associated with a young age of onset, high rate of lymphadenopathy and poor prognosis. Radical resection of the lung and systemic lymph nodes may provide complete relief from symptoms.

\section{Acknowledgements}

The current study was supported by the Natural Science Foundation of Liaoning Province (grant no. 2015020561) and the Fund for Scientific Research of The First Hospital of China Medical University (grant no. fsfh1514).

\section{References}

1. Wajchenberg BL, Mendonca BB, Liberman B, Pereira MA, Carneiro PC, Wakamatsu A and Kirschner MA: Ectopic adrenocorticotropic hormone syndrome. Endocr Rev 15: 752-787, 1994

2. Quaedvlieg PF, Visser O, Lamers CB, Janssen-Heijen ML and Taal BG: Epidemiology and survival in patients with carcinoid disease in the Netherlands. An epidemiological study with 2391 patients. Ann Oncol 12: 1295-1300, 2001.

3. Modlin IM, Lye KD and Kidd M: A 5-decade analysis of 13,715 carcinoid tumors. Cancer 97: 934-959, 2003.

4. Hemminki $\mathrm{K}$ and $\mathrm{Li} \mathrm{X}$ : Incidence trends and risk factors of carcinoid tumors: A nationwide epidemiologic study from Sweden. Cancer 92: 2204-2210, 2001.

5. Fink G, Krelbaum T, Yellin A, Bendayan D, Saute M, Glazer M and Kramer MR: Pulmonary carcinoid: Presentation, diagnosis, and outcome in 142 cases in Israel and review of 640 cases from the literature. Chest 119: 1647-1651, 2001.

6. Gatta G, Ciccolallo L, Kunkler I, Capocaccia R, Berrino F, Coleman MP, De Angelis R, Faivre J, Lutz JM, Martinez C, et al; EUROCARE Working Group: Survival from rare cancer in adults: A population-based study. Lancet Oncol 7: 132-140, 2006.

7. Yao JC, Hassan M, Phan A, Dagohoy C, Leary C, Mares JE, Abdalla EK, Fleming JB, Vauthey JN, Rashid A and Evans DB: One hundred years after 'carcinoid': Epidemiology of and prognostic factors for neuroendocrine tumors in 35,825 cases in the United States. J Clin Oncol 26: 3063-3072, 2008.
8. Zuetenhorst JM and Taal BG: Metastatic carcinoid tumors: A clinical review. Oncologist 10: 123-131, 2005.

9. Beasley MB, Brambilla E, and Travis WD: The 2004 World Health Organization classification of lung tumors. Semin Roentgenol 40: 90-97, 2005.

10. Boddaert G, Grand B, Le Pimpec-Barthes F, Cazes A, Bertagna $\mathrm{X}$ and Riquet M: Bronchial carcinoid tumors causing Cushing's syndrome: More aggressive behavior and the need for early diagnosis. Ann Thorac Surg 94: 1823-1829, 2012.

11. Amer KM, Ibrahim NB, Forrester-Wood CP, Saad RA and Scanlon M: Lung carcinoid related Cushing's syndrome: Report of three cases and review of the literature. Postgrad Med J 77: 464-467, 2001.

12. Terzolo M, Reimondo G, Ali A, Bovio S, Daffara F, Paccotti P and Angeli A: Ectopic ACTH syndrome: Molecular bases and clinical heterogeneity. Ann Oncol 12 (Suppl 2): S83-S87, 2001.

13. Arioglu E, Doppman J, Gomes M, Kleiner D, Mauro D, Barlow C and Papanicolaou DA: Cushing's syndrome caused by corticotropin secretion by pulmonary tumorlets. N Engl J Med 339: 883-886, 1998

14. Shrager JB, Wright CD, Wain JC, Torchiana DF, Grillo HC and Mathisen DJ: Bronchopulmonary carcinoid tumors associated with Cushing's syndrome: A more aggressive variant of typical carcinoid. J Thorac Cardiovasc Surg 114: 367-375, 1997.

15. Aniszewski JP, Young WF Jr, Thompson GB, Grant CS and van Heerden JA: Cushing syndrome due to ectopic adrenocorticotropic hormone secretion. World J Surg 25: 934-940, 2001.

16. Ilias I, Torpy DJ, Pacak K, Mullen N, Wesley RA and Nieman LK: Cushing's syndrome due to ectopic corticotropin secretion: Twenty years' experience at the National Institutes of Health. J Clin Endocrinol Metab 90: 4955-4962, 2005.

17. de Matos LL, Trufelli DC, das Neves-Pereira JC, Danel C and Riquet M: Cushing's syndrome secondary to bronchopulmonary carcinoid tumor: Report of two cases and literature review. Lung Cancer 53: 381-386, 2006.

18. Mansi L, Rambaldi PF, Panza N, Esposito D, Esposito V and Pastore V: Diagnosis and radioguided surgery with 111In-pentetreotide in a patient with paraneoplastic Cushing's syndrome due to a bronchial carcinoid. Eur J Endocrinol 137: 688-690, 1997.

19. Markou A, Manning P, Kaya B, Datta SN, Bomanji JB, and Conway GS: [18F]fluoro-2-deoxy-D-glucose ([18F]FDG) positron emission tomography imaging of thymic carcinoid tumor presenting with recurrent Cushing's syndrome. Eur J Endocrinol: 152: 521-525, 2005.

20. Zemskova MS, Gundabolu B, Sinaii N, Chen CC, Carrasquillo JA, Whatley M, Chowdhury I, Gharib AM and Nieman LK: Utility of various functional and anatomic imaging modalities for detection of ectopic adrenocorticotropin-secreting tumors. J Clin Endocrinol Metab 95: 1207-1219, 2010.

21. Gustafsson BI, Kidd M, Chan A, Malfertheiner MV and Modlin IM: Bronchopulmonary neuroendocrine tumors. Cancer 113: 5-21, 2008.

22. Kara M, Ucar I, Yazicioglu A and Firat P: An overlooked tumor of the lung in Cushing's syndrome: Adrenocorticotropic hormone-secreting carcinoid tumor. Thorac Cardiovasc Surg 55: 527-529, 2007.

23. Ejaz S, Vassilopoulou-Sellin R, Busaidy NL, Hu MI, Waguespack SG, Jimenez C, Ying AK, Cabanillas M, Abbara M and Habra MA: Cushing syndrome secondary to ectopic adrenocorticotropic hormone secretion: The University of Texas MD Anderson Cancer Center Experience. Cancer 117: 4381-4389, 2011.

24. Dimopoulos MA, Fernandez JF, Samaan NA, Holoye PY and Vassilopoulou-Sellin R: Paraneoplastic Cushing's syndrome as an adverse prognostic factor in patients who die early with small cell lung cancer. Cancer 69: 66-71, 1992.

25. Izzedine H, Besse B, Lazareth A, Bourry EF and Soria JC: Hypokalemia, metabolic alkalosis, and hypertension in a lung cancer patient. Kidney Int 76: 115-120, 2009.

26. Winquist EW, Laskey J, Crump M, Khamsi F and Shepherd FA: Ketoconazole in the management of paraneoplastic Cushing's syndrome secondary to ectopic adrenocorticotropin production. J Clin Oncol 13: 157-164, 1995.

27. Nagy-Mignotte H, Shestaeva O, Vignoud L, Guillem P, Ruckly S, Chabre O, Sakhri L, Duruisseaux M, Mousseau M, Timsit JF and Moro-Sibilot D; Multidisciplinary Thoracic Oncology Group at Grenoble University Hospital, France: Prognostic impact of paraneoplastic cushing's syndrome in small-cell lung cancer. J Thorac Oncol 9: 497-505, 2014 